\title{
The Current Situation and the Future of the Tuvan Language in Krasnoyarsk Region: Ethnopsycholinguistic Survey Results
}

\author{
Olga V. Felde, Anastasia V. Kolmogorova \\ and Tamara N. Zhuravel* \\ Siberian Federal University \\ 79 Svobodny, Krasnoyarsk, 660041, Russia
}

Received 16.11.2016, received in revised form 15.12.2016, accepted 11.01.2017

\begin{abstract}
The destiny of minority languages is a crucial problem of sociolinguistics. The main objective of our research is to assess subjective factors that may influence the development of an indigenous language in Krasnoyarsk Region, the language being the Tuvan language spoken in the basin of the river Us, a tributary of the Yenisei. The ethnopsycholinguistic method of open-ended statements, applied in our work with the Russians and the Tuvinians cohabitating on the territory, allows us to see the differences in their attitudes towards the current state and the future of the Tuvan language.

Generally speaking, the results show that the Tuvan population believes that their language is in the process of survival even while going through hard times. However, they feel uncertainty about the future of Tuvan as it may be at risk of becoming a language of family communication, a language that is totally irrelevant for the most of local society. On the contrary, the Russian population of the region is confident of the future of the Tuvan language spoken in the basin of the river Us. At the same time, unlike autochthonous people, the Russians find a current situation of Tuvan as a disastrous one.

Basing on the analysis of the mentioned above open-ended statements experiment results, we argue that the position of the Tuvan language is very fragile nowadays. Yet, it has good prospects provided that the Tuvan population is strongly supported by the authorities in their will to preserve their own language. The fact that the Russian population shares the Tuvans' concerns over the eventual reduction of the Tuvan language functioning on the territory can be also regarded as a supporting argument. The study of these opinions in the intergroup context gives some clues for predicting the changes in the linguistic landscape of the region.
\end{abstract}

Keywords: Tuvan, minority, ethnopsycholinguistic, attitudes, language policy.

DOI: 10.17516/1997-1370-0002.

Research area: philology.

(C) Siberian Federal University. All rights reserved

* Corresponding author E-mail address: zhuravel_petamal@mail.ru; nastiakol@mail.ru; feldeo@list.ru 


\title{
Le présent et l'avenir de la langue touvine dans \\ la région de Krasnoïarsk: \\ analyse des résultats d'une enquête \\ éthnopsycholinguistique
}

\author{
Olga V. Feldé, Anastasia V. Kolmogorova, \\ Tamara N. Zhuravel \\ Université fédérale de la Sibérie \\ 79 Svobodny, Krasnö̈arsk, 660041, Russie
}

\begin{abstract}
Le destin des langues minoritaires est un des problèmes cruciales dont la sociolinguistique s'occupe. L'objectif principal de nos recherches c'est l'évaluation des facteurs subjectifs qui pourront influer sur le développement d'une des langue autochtone de la région de Krasnoïarsk-le touvin dans le bassin de la rivière Ousse, un des affluents de l'Ienisseï. L'analyse des résultats de l'enquêtement auprès des colléctivités russe et touvine cohabitant sur le territoire nous permettent de voir les différences des attitudes des Russes et des Touvins envers l'état actuel et l'avenir de la langue touvine. L'étude de ces opinions dans le contexte intergroupe donne des pistes pour prévoir des changements dans le paysage linguistique de la région.
\end{abstract}

Mots-clés : touvin, minorité, ethnopsycholinguistique, attitudes, politique linguistique.

\section{Introduction}

La vitalité d'une langue minoritaire est sa caractéristique définitoire dont l'évaluation adéquate permet aux chercheurs de prévoir l'avenir de cette langue et, conformément à ces prévisions, de proposer des décisions valables pour aménager la situation linguistique sur le territoire de façon à ce qu'elle soit favorable à la langue minoritaire en question. Dans le cadre sociolinguistique, l'étude de la vitalité est toujours confrontée à deux problèmes. Premièrement, en se penchant sur la vitalité d'une langue minoritaire, les sociolinguistes sont plutôt enclins à prendre en considération des facteurs objectifs (soutien gouvernemental, statut officiel et légitime de la langue dans la région, sa présence dans l'espace social public en tant que langue de la presse régionale etc.) qui influent sur son potentiel vital en sous-estimant l'influence des facteurs subjectifs réflétant les attitudes de la population autochtone elle-même envers sa langue maternelle aussi bien que les opinions des collectivités de leurs voisins ethniques vivant sur le même territoire. Deuxièmement, si l'étude est tout de même axée sur l'analyse des attitudes subjectives il se pose toujours la question de l'outil méthodologique dont l'application permettra d'obtenir des résultats pertinents et libres de tout ce qui aurait pû être conventionnel, arbitraire ou situationnel.

Leprésent article est consacréàla description des résultats de notre travail expérimental effectué dans le bassin d'un des affluents de l'Ienisseï - celui de l'Ousse, où deux groupes ethniques, les Russes et les Touvins, cohabitent depuis des siècles. Nos recherches sont surtout centrées sur l'estimation de la vitalité de la langue touvine selon les opinions des populations russe et touvine qui vivent dans ce contexte intergroupe. La méthode que nous appliquons est empruntée à l'éthnoplycholingustique et elle consite à proposer aux personnes interrogées de développer des phrases dont le début est déjà proposé. 


\section{Surestimation du rôle}

\section{des facteurs objectifs dans l'évaluation}

de la vitalité d'une langue minoritaire

Les recherches dans le domaine de l'évaluation de la vitalité des langues dites « autochtones » sur le territoire russe sont considérées par les sociolinguistes du pays parmi les plus importantes et les plus urgentes. Cet intérêt est surtout porté aux langues autochtones de la région sibérienne où elles fonctionnent en contact permanent avec le russe qui est la langue majoritaire.

Actuellement, la sociolinguistique dispose d'une base empirique assez riche pour prévoir l'impact des facteurs objectifs sur le développement et l'état de plusieurs langues ethniques des régions d'Altaï, de Khakassie, de Touva, de Yakoutie, d'Extrême Nord, des régions de Krasnoïarsk et de Tioumen. Nombreuses sont déjà ces études qui explorent la problématique de la force communicative des langues minoritaires en Sibérie, leurs sphères d'emploi, les enjeux principaux de la politique linguistique régionale qui dans leur intégralité dessinent les contours de la situation linguistique en Sibérie. Les ouvrages de T.G. Borgoiakova

(Borgoiakova, 2001, 2002), A.A. Bourykine (Bourykine, 2007, 2007 a), N.B. Vakhtine (Vakhtine 2004, 2008) et d'autres ont fait un considérable apport à la description intégrale de ces facteurs objectifs qui conditionnent le degré de vitalité des langues en question. Parmi ces facteurs objectifs les chercheurs citent comme les plus puissants : l'état démographique du groupe et le soutien institutionnel des organismes d'Etat dont celui-ci jouit. En revanche, le linguiste estonien M. Ehala (Ehala, 2010, p.85) réduit l'impact de ces facteurs sur la vitalité d'une langue : "quelque soit la réalité, - écrit-il, - si les locuteurs pecoivent leur groupe comme celui qui n'a plus de perspectives, ils se comportent toujours de la même façon : ils changent de langue sans prêter aucune attention au total du groupe, à son statut ou aux autres facteurs objectifs". C'est pourquoi une autre question, celle de l'influence des tendances et des changements subjectifs intérieurs aux organismes éthniques sur la capacité de survie des langues autochtones, se pose mais ne trouve pas encore de réponse.

Cela paraît évident que la vitalité d'une langue et sa faculté au glissement linguistique sont reliés par des rapports d'opposition : plus la langue est vitale moins elle est susceptible d'être remplacée par la langue majoritaire. Mais cette évidence n'est que superficielle. Des études actuelles montrent que le choix de la langue que fait un groupe ethnique minoritaire dépend de facteurs multiples qui ne sont pas toujours accessibles à l'observation extérieure. Ce fait mène parfois à la situation où une langue classée comme tout à fait vitale selon les paramètres objectifs cède d'une façon rapide et inattendue ses fonctions communicatives primaires à la langue de la majorité ethnique. Le chercheur américain J. Fishman (Fishman 1971, p. 451) remarque à ce propos: "Plusieurs facteurs qui sont le plus souvent censés d'influer profondement sur les procéssus de la résistance d'une langue minoritaire ou de sa disparition, ces facteurs peuvent agir en directions opposées en fonction $\mathrm{du}$ contexte sociolinguistique et psychologique ou dans certaines circonstances ils peuvent être complétement nivelés". Ainsi, le risque et la vitesse du " glissement linguistique”, c'est-à-dire de la substitution progressive de la langue de la majorité ethnique à celle de la minorité, semble être étroitement lié aux attitudes des autochtones envers leur langue maternelle, qui est celle de leurs ancêtres.

Tout de même, rares encore sont les travaux qui accordent une attention particulière à l'évaluation des perceptions, des attitudes subjectives, des opinions des représentants des groupes minoritaires envers la langue de leurs 
ancêtres dans le contexte intergroupe. Le premier questionnaire de vitalité subjective (Bourhis, Giles, Rosenthal, 1981) a été élaboré et mis en place en 1981. Un peu plus tard, en 1987 R. Allard et R.Landry (Alard, Landry 1987) ont conçu la notion de « croyance » qui permettrait d'englober ou d'accommoder les concepts de perception, attitude, valeur, opinion, auto-évaluation etc., qui sont présents dans divers modèles et théories en psychologie sociale du langage. Les huit croyances proposées par ces chercheurs étaient reflétées dans leur questionnaire de vitalité subgective. En tant que croyances cruciales à évaluer le luinguiste bouriate E.B. Khikhlanova (Khikhlanova, 2007) cite les appréciations, les dispositions cognitivo-affectives dans lesquelles la langue maternelle est saillante. L'étude de ces dispositions aiderait à prédire le comportement langagier des locuteurs autochtones dans les milieux où deux communautés ethnolinguistiques sont en contact.

Ainsi, dans notre recherche nous nous donnons pour but de décrire l'ensemble des facteurs subjectifs qui pourraient influer sur la vitalité de telle langue minoritaire de la région de Krasnoïarsk comme celle de la langue touvine sur le territoire du bassin de la rivière Ousse (Oussinskaja kotlovina) .

\section{Langue touvine dans la vallée de l'Ousse : description de la situation linguistique existante}

La langue touvine qui est l'objet de nos études est la langue maternelle pour 1500 Touvins habitant la région de Krasnoïarsk. Il est à noter que la population touvine y reste stable depuis déjà 40 ans. La plus grande part des Touvins (environ 500 personnes) habitent à Krasnoïarsk, la capitale de la région; ce sont principalement des jeunes qui y font leurs études. Encore 400 personnes vivent dans la commune Ermakovskij, territoire frontalier avec la république de
Touva. Le taux de ceux qui parlent leur langue maternelle est très élévé - 92, $6 \%$. Au Sud de la région de Krasnoïarsk, dans le bassin de la rivière Ousse, un des affluents de l'Ienisseï, les communautés touvines sont concentrées dans les villages Verkhneoussinskoje, Nignéoussinskoje, Oust'-Zolotaja et Maralsovkoz. Ce territoire est traditionnellement habité par les Russes et les Touvins depuis le XIX ${ }^{\text {e }}$ siècle mais, selon les données de l'historien de la région V.G. Datsichène (Datsichène 2009), leur métissage n'a commencé qu'au début du $\mathrm{XX}{ }^{\mathrm{e}}$ sciècle. A partir de ce moment la situation change globalement ce qui est dû à la politique linguistique de l'URSS concernant les Touvins du bassin de l'Ousse. Cette politique ne visait pas à imposer le russe comme la seule langue de communication mais se donnait pour but de créer les meilleures conditions pour le développement du bilinguisme dans la région. La promotion du bilinguisme a débuté avant tout dans le domaine de l'enseignement public. Au village Verkhneoussinskoje le premier internat a été organisé pour les enfants touvins où on enseignait deux langues : le russe et le touvin.

Jusqu'aux années 2000 la situation bilingue dans le bassin de l'Ousse paraîssait assez stable et équilibrée. En revanche, le paysage sociolinguistique actuel montre des changements profonds intervenant au fil du temps parmi lesquels trois sont à signaler : la banalisation des mariages mixtes, la diminution du rôle des cultes religieux pour les Russes aussi bien que pour les Touvins et, enfin, la dégradation progressive de la langue touvine dans le système d'enseignement public régional. La seule et l'unique sphère où le touvin résiste encore en conservant ses fonctions et son importance d'autrefois est la communication quotidienne dans le cadre familial.

Dans le contexte décrit, la vitalité de la langue touvine dépend en grande partie de la volonté des Touvins du bassin de l'Ousse de préserver leur langue, de la parler et de la transmettre à leurs 
enfants comme partie du patrimoine national, c'est-à-dire que la vitalité du touvin est étroitemnt liée aux facteurs subjectifs dont l'évaluation se présente comme une entreprise de grande urgence.

\section{Organisation et résultats de l'application de la méthode ethnopsycholinguistique}

Dans le cadre de l'approche sociolinguistique l'enquête et l'interview sont traditionnellement considérés comme les outils-clé pour une étude de la vitalité d'une langue. Mais nous ne pouvons pas négliger le fait que les réponses des personnes interrogées sont tributaires des attitudes pragmatiques et situationnelles : essayer de deviner les attentes des chercheurs en leur fournissant des réponses "correctes", conformes aux idées reçues des personnes interrogées sur ce qui est attendu d'elles.

Afin de contourner ce biais nous avons proposé aux Touvins et aux Russes du bassin de l'Ousse de développer quelques phrases dont les débuts étaient : "A présent la langue des Touvins.... » et « Dans l'avenir la langue des Touvins....".

Une telle consigne, élaborée conformément aux traditions de la méthodologie ethnopsycholinguistique et connue comme la méthode des phrases à développer, n'expose pas la personne interrogée aux risques de formuler son attitude envers la langue en général, mais, par contre, l'invite à évaluer la position actuelle de la langue en question dans la vie de la région et à proposer sa vision du statut de la langue dans les années à venir. Soulignons que nous estimons crucial de connaître l'opinion des deux collectivités ethniques cohabitant depuis des sciècles dans le bassin de l'Ousse - russe et touvine - afin de se faire une image complète et détaillée des facteurs subjectifs suseptibles d'influencer la vitalité de la langue touvine.
Au cours des enquêtes effectuées en 2011 au village Verkhneoussinskoje nous avons reçu 121 réactions dont 43 étaient données par des Touvins et 78 par des Russes. La différence quantitative entre les représentants de deux groupes ethniques qui ont participés à l'enquête s'explique par l'importance des diasporas dans la population de Verkhneoussinskoje. Toutes les réponses ont été réparties en 3 groupes selon leur tonalité axiologique : attitudes négatives, attitudes neutres et attitudes positives. Les résultats sont présentés dans les grilles ci-dessous.

L'analyse des opinions subjectives des autochtones envers les perspectives de leur langue montre que ceux-ci envisagent son avenir avec une certaine prudence ou expriment sentiment pessimiste : les attitudes négatives dépassent plus qu'en moitié celles qui sont positives ou neutres. Tandis que la situation actuelle du touvin dans la région se présente aux locuteurs natifs en couleurs moins sombres : les opinions se sont réparties en proportions presque égales avec une légère prédominance des attitudes positives.

Les sujets russes sont enclins à constater la perte par le touvin de ses positions dans le paysage linguistique régional du bassin de l'Ousse, mais en revanche, ils ne croient pas à son déclin définitif dans les années à venir : seulement 18 sur 27 ne voient pas de perspectives pour la langue de leurs voisins ethniques tandis que 15 de ces 27 sont persuadés que le touvin surmontera toutes les difficultés et restera viable.

Dans un second temps, à chaque catégorie de réponses nous pouvons distinguer encore quelques sous-groupes où les nuances subjectives se prononcent différemment. Ainsi, en analysant les attitudes négatives des Russes et des Touvins, deux groupes ethniques mélangés, envers les perspectives du touvin nous constatons deux tendances générales qui ne dépendent pas de l'appartenance ethnique des personnes interrogées : 1) il y a ceux qui croient que la disparition de la 
Tableau. 1. Attitudes des Touvins envers la position de la langue touvine sur les deux plans temporels

\begin{tabular}{|l|c|c|c|}
\hline & Attitudes négatives & Attitudes neutres & Attitudes positives \\
\hline Position de la langue touvine à présent & 6 & 7 & 8 \\
\hline Position de la langue touvine dans l'avenir & 12 & 5 & 5 \\
\hline
\end{tabular}

Tableau. 2. Attitudes des Russes envers la position de la langue touvine sur les deux plans temporels

\begin{tabular}{|l|c|c|c|}
\hline & Attitudes négatives & Attitudes neutres & Attitudes positives \\
\hline Position de la langue touvine à présent & 21 & 19 & 3 \\
\hline Position de la langue touvine dans l'avenir & 18 & 12 & 15 \\
\hline
\end{tabular}

langue touvine de la carte linguistique du bassin de l'Ousse sera complète et inévitable ; 2) il y a ceux qui ne prévoient qu'une certaine restriction des fonctions du touvin mais ne croient pas à sa mort définitive. Comme exemple du premier type nous pouvons citer l'opinion d'un des sujets qui a écrit : "Dans l'avenir la langue touvine disparaîtra à jamais". En guise d'exemple du deuxième type citons les deux opinions suivantes : "Dans l'avenir la langue touvine ne sera plus enseignée dans les écoles du bassin de l'Ousse" et "Dans l'avenir la langue touvine deviendra une langue rare". Les Russes et les Touvins expliquent leurs prévisions négatives par le fait que les Touvins sont de moins en moins motivés pour parler leur langue entre eux, dans leur famille ou avec leurs enfants. Il est surtout à noter que les attitudes extrêmement négatives sont beaucoup plus nombreuses que celles où le négativisme ne semble pas absolu, c'est-à-dire parmi les sujets « pessimistes » quant à l'avenir du touvin, le nombre de ceux qui prédisent l'extinction du touvin dépasse celui de ceux qui parlent seulement de la restriction de ces fonctions.

Quant à l'état actuel du touvin dans le bassin de l'Ousse, les autochtones semblent, généralement, plus optimistes que les Russes. Mais les deux collectivités ethniques relient l'affaiblissement du touvin en tant que langue au fait qu'elle n'est plus enseignée dans toutes les écoles de la commune de Ermakovskij, territoire principalement habité par les Touvins. En même temps, ce fait est différemment traité par les Russes et les Touvins. Pour les premiers, une langue qui n'est plus incorporée aux programmes scolaires est sûrement condamnée à mort, pour les seconds, locuteurs natifs de la langue en question, le touvin, même lorsqu'il se trouve hors le système scolaire, semble assez viable grâce à son fonctionnement au sein des familles comme langue de communication quotidienne. Aussi les Touvins du bassin de l'Ousse, même classés du côté des "pessimistes", ne voient pas de menace directe contre leur langue maternelle, ce qui se conjuge difficilement avec leurs prédictions tristes concernant l'avenir de leur langue.

Les attitudes neutres prédominent chez les représentants de toutes les deux collectivités ethniques dans les deux plans temporels. En général, ce sont des phrases où l'on constate un certain état des choses, où l'on ne fait que citer des faits sans tenter de les intégrer dans une quelconque vision plus subjective. Des exemples à citer : "le touvin c'est la langue maternelles des Touvins", "le touvin n'est plus enseigné dans les écoles de la commune". Dans la même catégorie des attitudes neutres nous avons aussi classé des phrases contenant l'opinion des Russes sur l'importance du touvin pour eux personellement: 
«le touvin, je n'en ai pas besoin », «le touvin n'a pas d'importance pour moi », en les estimant trop centrées sur l'individu et non sur le positionnemnet de la langue. Des obligations morales dépersonnalisées comme "le touvin doit s'épanouir et les Touvins en quittant leur région ne doivent pas oublier leur langue» ont été, elles aussi, classées dans la catégorie des «neutres ».

Dans le cas des attitudes positives concernant la force et la vitalité du touvin à l'heure actuelle, les autochtones soulignent le fait important que tous les représentants de la collectivité touvine dans la région parlent leur langue maternelle, par exemple : «à présent le touvin est parlé par tous les Touvins de la région », « en ce moment tous, enfants et grandes personnes, parlent leur langue» etc. Parmi les rares réponses positives des Russes nous remarquons des idées analogues.

L'avenir du touvin se présente sans nuages pour la minorité des personnes interrogées : c'est le cas de 5 Touvins sur 43 qui ont participé à l'enquête et de 15 Russes sur 78 au total. Il est surprenant de constater que ce sont uniquement les Touvins qui dessinent des images fantastiques du futur succès de leur langue de sorte qu'elle « soit la langue la plus répandue au monde » ou « qu'elle soit la langue la plus parlée en Russie et dans le monde entier ». Parmi ces phrases dont l'intention encourageante les tranforme plutôt en formules magiques, en souhaits irréalisables mais très désirés, on retrouve seulement une projection qui ressemble à la vérité optimiste et un avertissement à la fois : « Dans l'avenir le touvin existera toujours. Si nous n'avons plus notre langue, nous ne serons plus une nation ». Les Russes espèrent vivement que le touvin reprendra ses fonctions perdues au cours des années (« dans l'avenir le touvin retrouvera sa place dans l'enseignement public de la région », " dans l'avenir tous les Touvins parleront leur langue ») et que son importance ne va faire qu'augmenter chaque année (« dans l'avenir la langue touvine sera très importante dans la région »).

\section{Conclusion}

L'analyse comparative des attitudes subjectives des représentants de deux collectivités ethniques (russe et touvines) envers la vitalité de la langue touvine dans le bassin de l'affluent de l'Ienisseï qu'est la rivière Ousse montre qu'en général, la population russe croit à la possibilité de conserver la langue touvine et même de la développer et de réaffirmer son importance dans le bassin de l'Ousse. À l'inverse, la population touvine de la région manifeste son découragement dû à la situation du touvin qui n'est plus enseigné dans les écoles de la commune et se cantonne actuellement à la communication quotidienne et familiale. Il est important que la population autochtone souhaite ardemment conserver sa langue maternelle, ce qui témoigne du potentiel considérable de la vitalité que cette langue possède. Mais ce potentiel n'est réalisable qu'à condition que les Touvins encouragés et soutenus par les pouvoirs locaux puissent s'investir personellement et socialement dans l'organisation progressive de stuctures telles que des sociétés, des associations et autres groupes visant à faire revenir le touvin dans le système scolaire, dans la presse régionale et dans la littérature nationale. Cette réaffirmation de la langue touvine dans la région $\mathrm{du}$ bassin de l'Ousse est tellement attendue, d'autant plus que les conditions objectives et subjectives demeurent très favorables.

\section{References}

Allard, R., Landry, R. (1987). Contact des langues, vitalité ethnolinguistique subjective et comportement langagier, In Langage et société, 41, 83-84. 
Borgoiakova, T.G. (2001). Minoritarnye iazyki: problemy sokhraneniia i razvitiia [Minority Languages: Problems of Conservation and Development]. Abakan, HGU, 159 p.

Borgoiakova, T.G. (2002). Sotsiolingvisticheskie protsessy v respublikah Iuzhnoi Sibiri [Sociolinguistic Pprocesses in the Republics of Southern Siberia]. Abakan, Ros. akademiia nauk, In-t iazykoznaniia, Hakasskii gos. un-t im. N.F. Katanova; Institut iazykoznaniia, 264 p.

Burykin, A.A. (2007). Korennye narody Tiumenskoi oblasti i nekotorye obshchie problemy izucheniia kul'tur i sotsiumov korennogo naseleniia v polietnichnykh regionakh [Indigenous Peoples of Tiumen' Region and Some General Problems of Indigenous Cultures and Societies Study in Multy-Ethnic Territories], Kul'turnoe nasledie Tiumenskoi oblasti: materialy nauchno-prakticheskoi konferentsii "Kul'turogenez $i$ problemy aktualizatsii kul'turnogo naslediia narodov Tiumenskoi oblasti" [Cultural Heritage of Tiumen' Region: Proceedings of the International Conference "The Genesis of Culture and Problems of Tiumen' Region Cultural Heritage Actualization], Tiumen, RIC TGAKI, 5-8.

Burykin, A.A. (2007a). Regional'naia spetsifika iazykovoi situatsii v subiektakh Rossiiskoi Federatsii [The Specificity of Linguistic Situation in the Russian Federation Regions]. Problemy dvuiazychiia (mnogoiazychiia) v sovremennom sotsiokul'turnom prostranstve: Materialy nauchnoprakticheskoi konferentsii [Proc. of the Conference "Problems of Bilingualism in its Social and Cultural Dimensions], Yakutsk, 119-131.

Bourhis, R.Y., Giles, H., Rosenthal, D. (1981). Notes on Construction of a 'Subjective Vitality Questionnaire' for Ethnolinguistic Groups, In Development, 2, 145-155.

Datsyshen, V.G. (2009). Tuvinskoe naselenie Usinskogo pogranichnogo okruga Eniseiskoi gubernii. Iz istorii russko-tuvinskikh otnoshenii [The Tuvan Heritage of the Border of the Us District of Yeniseisk Region], In Novye issledovaniia Tuvy [Modern Researches on Tuva Region], 3, available at: http://www.tuva.asia/journal/issue_3/465-dasishen.html

Ehala, M. (2010). Refining the Notion of Ethnolinguistic Vitality, In International Journal of Multilingualism, 7, 363-378.

Fishman, J.A. (1971). The Sociology of Language, In Advances in the Sociology of Language, 1, $450-475$.

Khilkhanova, I.B. (2007). Faktory kollektivnogo vybora iazyka i etnokul'turnaia identichnost' u sovremennykh buriat [Factors of Collective Language Choice, Ethnic and Cultural Identity of the Buriat People]. Ulan-Ude, 205 p.

Vakhtin, N.B. (2001). Usloviia iazykovogo sdviga (K opisaniiu sovremennoi iazykovoi situatsii na Krainem Severe) [Conditions of Language Glide (Towards a Description of Linguistic Situation in the Far North], In Vestnik molodykh uchenykh. Seriia: Filologicheskie nauki [Bulletin of Young Researchers. Series of Philological Sciences], 1, 11-16.

Vakhtin, N.B. (2004). Iazyki narodov Krainego Severa [Languages of the Far North peoples], In Vestnik Rossiiskoi Akademii Nauk [Herald of the Russian Academy of Sciences], 74 (4), 301-309. 


\section{Настоящее и будущее тувинского языка \\ в Красноярском крае: анализ результатов \\ этнопсихолингвистического эксперимента}

О.В. Фельде, А.В. Колмогорова, Т.Н. Журавель

Сибирский федеральный университет Россия, 660041, Красноярск, пр. Свободный, 79

В статье рассматривается проблема витальности миноритарного тувинского языка, функционирующего в условиях тесного контакта с русским. Анализ субъективной оценки жизнеспособности тувинского языка дается на материале психолингвистического эксперимента, который был проведен в населенных пунктах Усинской котловины Красноярского края.

Ключевые слова: миноритарный язык, мажоритарный язык, витальность, языковая ситуации, языковая политика, языковой сдвиг, психолингвистический эксперимент.

Научная специальность: 10.00.00 - филологические науки. 